\title{
Editorial
}

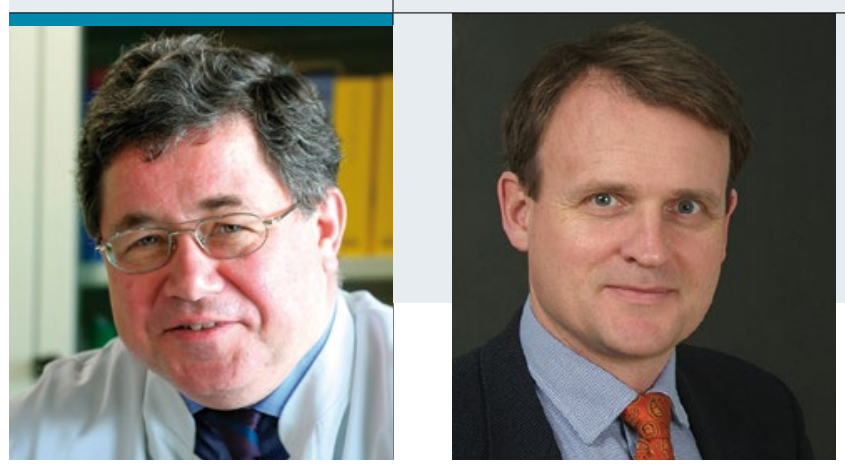

"Man muss bedenken, dass eine hohe

Wirksamkeit mit geringer Evidenz

und eine niedrige Wirksamkeit mit

hoher Evidenz bestehen kann."

Prof. Dr. Hans F. Merk, Hautklinik der Medizinischen Fakultät, Universitätsklinikum der RWTH Aachen

Prof. Dr. Thilo Jakob, Klinik für Dermatologie und Allergologie, Universitätsklinikum Gießen und Marburg, Standort Gießen

\section{Vorsicht! Verwirrspiel bei SIT-Präparaten}

$\mathrm{N}$ ach Publikation der jüngsten Leitlinie zur spezifischen Immuntherapie (SIT) werden nun unterschiedliche Tabellen mit Einteilungen der SIT-Präparate vorgelegt, die bei Verwendung verschiedener Einteilungskriterien zu einem allgemeinen Verwirrspiel ausarten. Den Vogel hat jetzt Ökotest abgeschossen. Unter der Überschrift „Happy Нуро“ - garniert mit Anzeigen, z. B. für das Heuschnupfenmittel DHU: „Heuschnupfen? Einfach wegpusten!“ - werden SIT-Präparate auf der Basis ihrer Fachinformationen und durch eine Bewertung nach Inhaltsstoffen eingeteilt. Enthält ein Präparat beispielsweise Phenol erhält es einen Malus. Auch Aluminiumhydroxid wird diskutiert. Hat der Hersteller jedoch die Konzentration für das Präparat angegeben, wird kein Malus vergeben, während ein Präparat ohne Angabe der Konzentration einen Malus erhält. Aus dem Ganzen ergibt sich eine starke Bewertung solcher Inhaltsstoffe, die in Schulnoten von „sehr gut“ bis „ohne Bewertung“ ausgedrückt wird. Bei fehlendem Phenol und Aluminium sind Tabletten und sublinguale Immuntherapie (SLIT) „sehr gut und alles andere "gut" bis „ohne Bewertung“.

$\mathrm{Zu}$ einem ganz anderen Ergebnis kommen in der aktuellen JACI-Ausgabe Stephen Durham und Martin Penagos bei einem Vergleich von SLIT und subkutaner Immuntherapie (SCIT) unter Berücksichtigung verschiedener Studienergebnisse auf Cochrane-Basis. Sie kommen im Ergebnis zu einer gleichwertigen Balance (SCIT: Wirksamkeit: +++; Sicher heit: +; SLIT: Wirksamkeit: ++; Sicherheit: ++) und empfehlen die Entscheidung zwischen SLIT und SCIT von der lokalen Verfügbarkeit und der per. sönlichen Bevorzugung von Arzt und Patient ab hängig zu machen [Durham SR, Penagos M. J Allergy Clin Immunol 2016;137:339-349.e10].

Das von Ökotest jetzt auf die Spitze getriebene Verwirrspiel mit unterschiedlichen Einteilungskri- terien besteht auch darin, Evidenz an die Stelle des Wirksamkeitsnachweises zu setzen. Dabei muss man bedenken, dass eine hohe Wirksamkeit mit geringer Evidenz, eine niedrige Wirksamkeit mit hoher Evidenz bestehen kann, bzw. je nach dem wie die Kriterien für den Wirksamkeitsnachweis festgelegt wurden, kann man hohe oder niederige Evidenz erwarten. Das Marketing kann sich dann aussuchen, was es jeweils bevorzugt. Rechtzeitig zu Beginn der Heuschnupfen-Saison erscheint daher in diesem Heft ein Positionspapier von Brehler et al., in dem die notwendigen Begriffe definiert werden. Das Positionspapier schafft damit eine Basis, um zusammen mit der SIT-Leitlinie eine vernünftige Auswahl der im Einzelfall richtigen SIT-Strategie zu treffen.

Das $\alpha$-Gal-Syndrom hat wichtige neue grundlegende Erkenntnisse zu allergischen Reaktionen wie verzögerte Sofortreaktionen und allergene Bedeutung von Kohlenhydraten gebracht. Hierzu finden Sie im aktuellen Heft thematisch gebündelt drei Übersichten von namhaften Experten aus den USA, Schweden und Deutschland. Damit nicht der Eindruck entsteht, dass jede allergische Reaktion auf Fleisch dem $\alpha$-Gal-Syndrom entspricht, werden in der nächsten Ausgabe zwei Übersichtsbeiträge die Fleischallergien, die a-Gal unabhängig sind, behandeln und damit den Themenschwerpunkt abrunden. Wir wünschen Ihnen viel Freude bei der Lektüre der aktuellen Ausgabe.

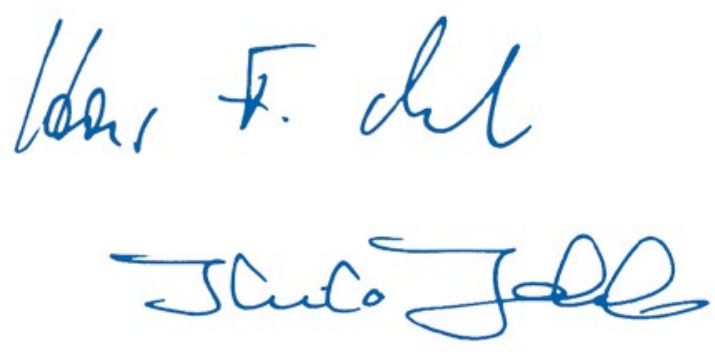

